\title{
Performance Analysis of STBC MIMO -OFDM Based WI-MAX for Various Equalizers to Reduce BER
}

\author{
Owk. Srinivasulu ${ }^{1,}$ Prof .P. Rajesh Kumar ${ }^{2}$, \\ ${ }^{1}$ Associate Professor, St. Peter's Engineering College-Hyd. Research Scholar, AUCE, Visakapatnam-03, \\ ${ }^{2}$ Professor\& Head Of The Department, Department Of ECE, AUCE, Visakapatnam-03
}

\begin{abstract}
Due to continually emerging demands of multimedia and internet services, in current years broadband wireless communication has gained attention. Wireless Communication mainly facing the main tasks of availability of resources like Bandwidth and transmission power, and also suffering from the problems of channel damages like fading and interference. Those problems can be avoided by using the Multiple Input Multiple Output (MIMO) and Orthogonal Frequency Division Multiplexing (OFDM) techniques. To increase the channel capacity and to maintain the high data rate mixture of (MIMO-OFDM) is implemented to offer a simple and high performance of the communication system. OFDM avoids inter-symbol interference (ISI) which is arise by synchronization error that degrades the signal performance. Parallel transmission is possible by OFDM and it uses the Wireless Interoperability for Microwave Access (WIMAX) on its physical layer. In adaptable WIMAX systems, MIMO techniques are prominent part in IEEE802.16e 2005 specifications. The objective of this paper is to reduce the bit error rate (BER) by placing the various equalizers at the receiver end simulation results verified by using MATLAB software.
\end{abstract}

Keywords: MIMO, OFDM, STBC, WIMAX, BER

\section{Introduction}

The main goal of the article is how to reduce the bit error rate with the help of various equalizers. Due to the easiness of use and mobility, wireless communication systems gain popularity, first these were designed for the sustenance of voice, later used for transmission of the data so playing the important part in this world. The major complications of wireless communications are inadequacy of communication resources, signal fading, multipath fading, and interference increase. In broad band wireless communications receiver complexity is reduced by OFDM but at same time channel estimation and synchronization are very important so it changed by MIMO-OFDM.MIMO_OFDM is good-looking equipment which generally combines on the same channel by permitting multiple accesses for wireless communication systems like Wireless Local Area Network, MAN, WiMAX and 3G-LTE standard in order to lodge many users at the same time in the same channel. Space time coding alludes channel coding strategy for transmissions with various transmit and get reception apparatuses. With the help of OFDM we can convert the main carrier in to closely spaced sub carriers there by inter symbol interference can be avoided and channel robustness can be improved. In connection with MIMO multiplexing gain spectral efficiency is possible. STBCs are basic, however viable instrument to get transmit assorted qualities in MIMO correspondence channel. Orthogonal STBCs intended for more than two radio wires can accomplish full differing qualities arrange however they have code rate not as much as solidarity. It is realized that the BER execution of orthogonal STBCs can be basically gotten from existing investigative outcomes for get assorted qualities maximal proportion consolidating (MRC) with a similar differing qualities arrange by fittingly scaling the SNR. In this paper, we reproduce and think about the BER execution of STBC OFDM framework utilizing 16 QAM regulation plans for SISO, MISO, MIMO (2X2) reception apparatus conspires over Rayleigh Channels. The mix of STBC and OFDM gives twofold orthogonally to the conveying signal and enhances security and broadcast of information.

\section{Literature Survey}

MIMO with OFDM reduces the equalization complexities by transmitting different data on different frequency levels to gain spectral efficiency and error recovery features, which will offer high spatial rate by transmitting data on multiple antennas and transmission in Non-Line-of sight (NLOS). Thus the MIMO-OFDM technique is used to achieve diversity. It will utilize the three basic parameters that is frequency (OFDM), time (STC) and spatial (MIMO). The MIMO-OFDM is the reproductive and highly famous services for Wireless broad band access. The combination of MIMO and OFDM accumulates the purpose of each and every scheme that will provide the high throughput. The current and main application of MIMO-OFDM is IEEE 802.16 (WiMAX) which will gain high reputation and the scholar's desirability for additional growth and improvement. This paper represents a facet summary of an analysis of MIMO-OFDM technique and its combination with 
Space Time Coding scheme, which reflects to most recent work of IEEE and Wi-MAX forum. To validate the solutions for the challenges identified through literary survey simulations will be carried out using Mat lab Simulator. The STC with MIMO systems is organized to get transmit range and allowing the secure means of propagation of data in scenario where mobility is required for such data transmission. At the receiver end full capacity gain and more signal to noise ratio achieved by maintaining the complete channel state information. The model outcomes are performed for different order of MIMO systems.

\section{Design Methodology}

MIMO_OFDM is very important combination in present and upcoming wireless communication systems and maintains robustness and high spectral efficiency by syndicates with MIMO which increases capacity by communicating different signals over multiple antennas and OFDM which divides the main carrier in to closely spaced subcarriers to deliver additional steadfast communication at high speed. It provides the highest capacity and data throughput. For wireless communications MIMO equipment is the major method which is engaged to increase the signal to noise ratio and link capacity. Even in rich scattering environment of modern wireless system design spectral efficiency gains, high signal quality and coverage by employing spatial diversity through the Multiple-Input Multiple-Output (MIMO) communications are possible. And other benefits of MIMO scheme are high signal quality, beam forming, and space time coding and interference cancellation. The capacity is proportional to the rank of MIMO channel. However, all the gains cannot be achieved simultaneously due to their dependence on antenna configuration and scattering environment. By using OFDM(i.e. multicarrier modulation scheme) not only we can avoid inter symbol interference also it increases communication channel capacity and it is rightly suitable for MIMO channels transmission so it is very important technology in broad band wireless communications and maintains simple equalization. The wanted necessities of communication are fulfilled by the mixture of MIMO-OFDM scheme, such as good analysis in NLOS atmosphere, trustworthy broadcast, high peak data rates as well as high spectral efficiency.

Proposed system is shown in below Fig 1. like the single antenna orthogonal frequency division multiplexing transmitter of MIMO-OFDM has $N t$ parallel transmission paths, every subdivision carrying out serial-to- parallel conversion, pilot insertion, $N$-point IFFT and cyclic extension before the TX signals are upconverted to RF and transmitted.it is important to note that to all $\mathrm{Nt}$ twigs digital modulation, channel encoding (i.e. for error correction and detection) and spatial multiplexing are applied. The receiver receives the transmitted signal first it should estimate and correct the possible symbol timing error and frequency subsets. Then, cyclic prefix is detached and $N$-point FFT is done for each receiver subdivision. We proposed the channel estimation algorithm based on single carrier processing that implies MIMO detection has to be done per OFDM subcarrier. Finally all the input binary data are recovered with certain BER.

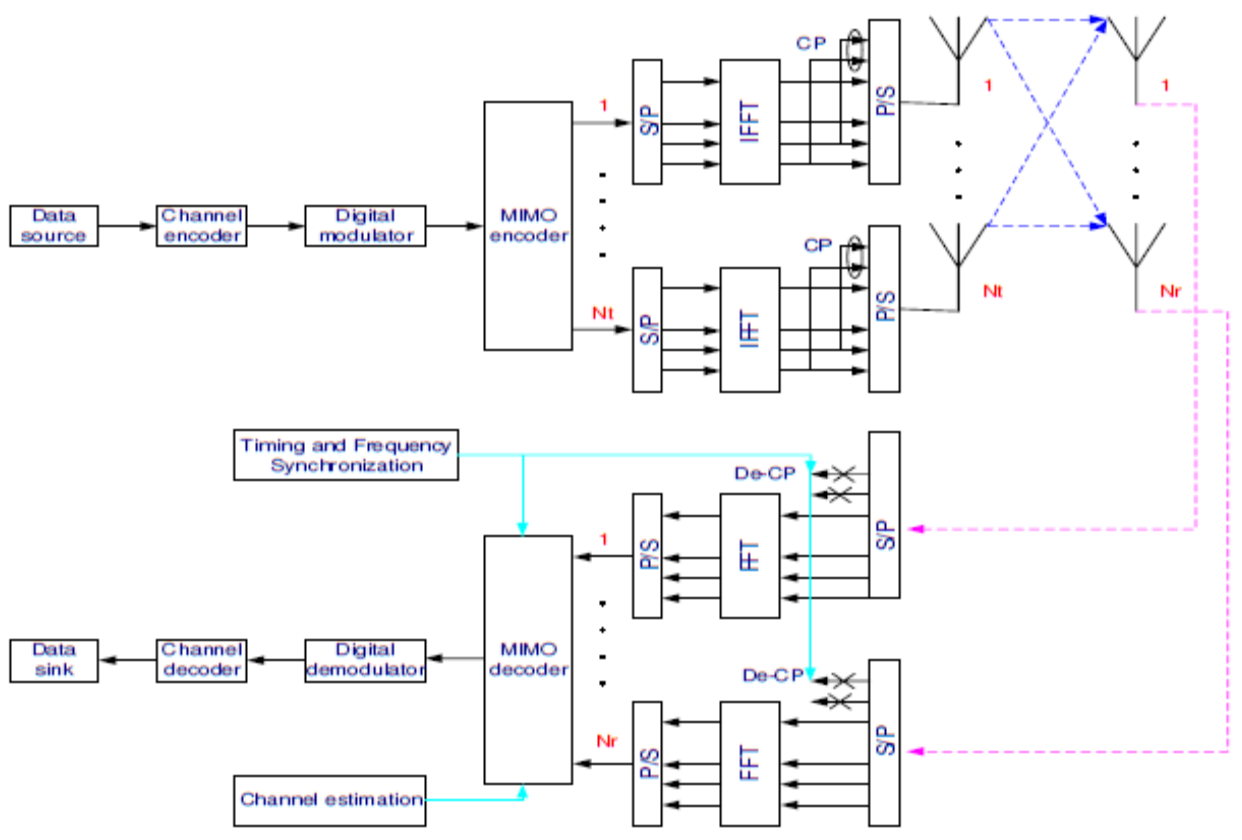

Fig 1.Proposed model 
A. Space-Time Block Coding (STBC): In 1998 Siavash Alamouti proposed a two antenna STBC that does not require any bandwidth expansion at the transmitter. His two antenna scheme was generalized to incorporate more antennas and thus increasing the block size operated on, however, the two antenna transmit scheme is a rate 1 code and it has been shown that this is the only configuration to achieve this rate. Here we look at the case presented in for two transmit antennas. The Alamouti coded OFDM system is shown as below. As before, we consider the Rayleigh multipath fading channel and a delay spread less than the cyclic prefix length. It is important to remember that using OFDM as a modulation scheme allows the assumption of a flat fading channel for each sub-carrier. By using space time coding in wireless communication systems data transmission reliability can maintained in connection with multiple transmit antennas.

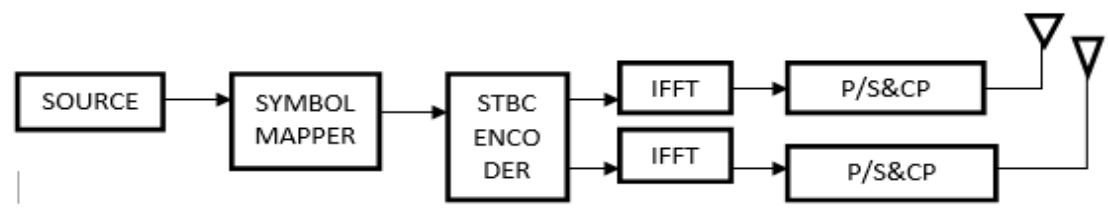

Fig 2. Alamouti for Two Transmit Antennas

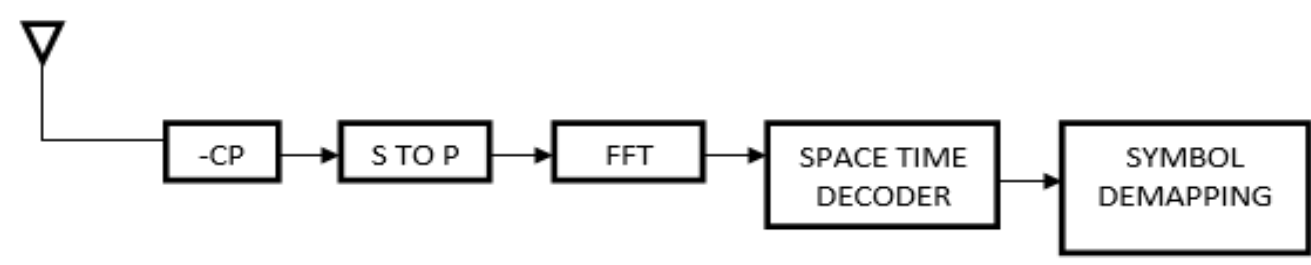

Fig 3. Altamonte for One Receiver Antenna

B. Equalizers: when the signals are passed through the channel, noise and distortion changes the signal characteristics and degrades the signal performance, here we need the equalizers to bring back the original signal characteristics. The basic block diagram of equalization circuit and structure of equalization are shown below Fig 4 and Fig 5.

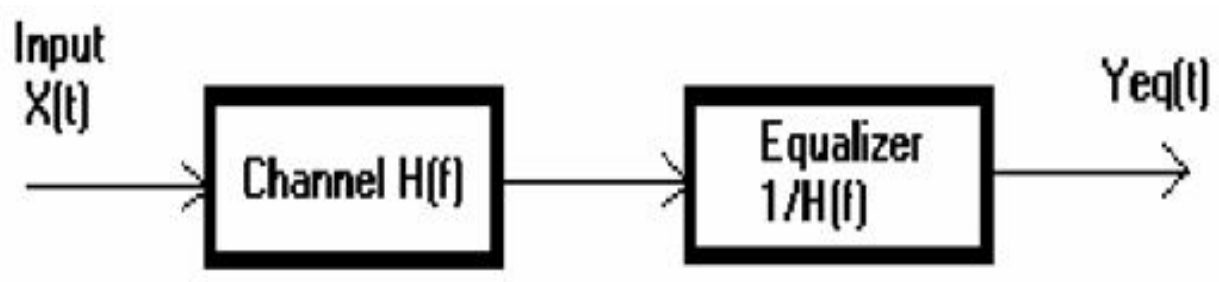

Fig 4. Basic Diagram of Equalization

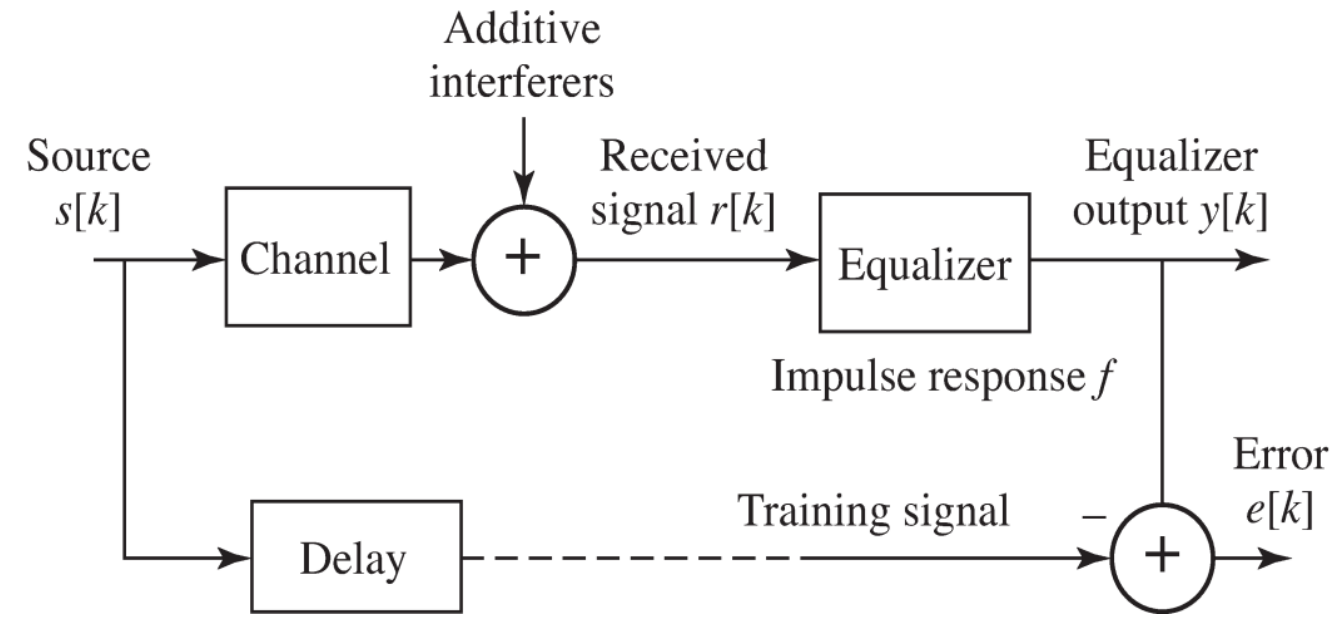


Fig 5. Structure of Equalization

\section{Simulation Results}

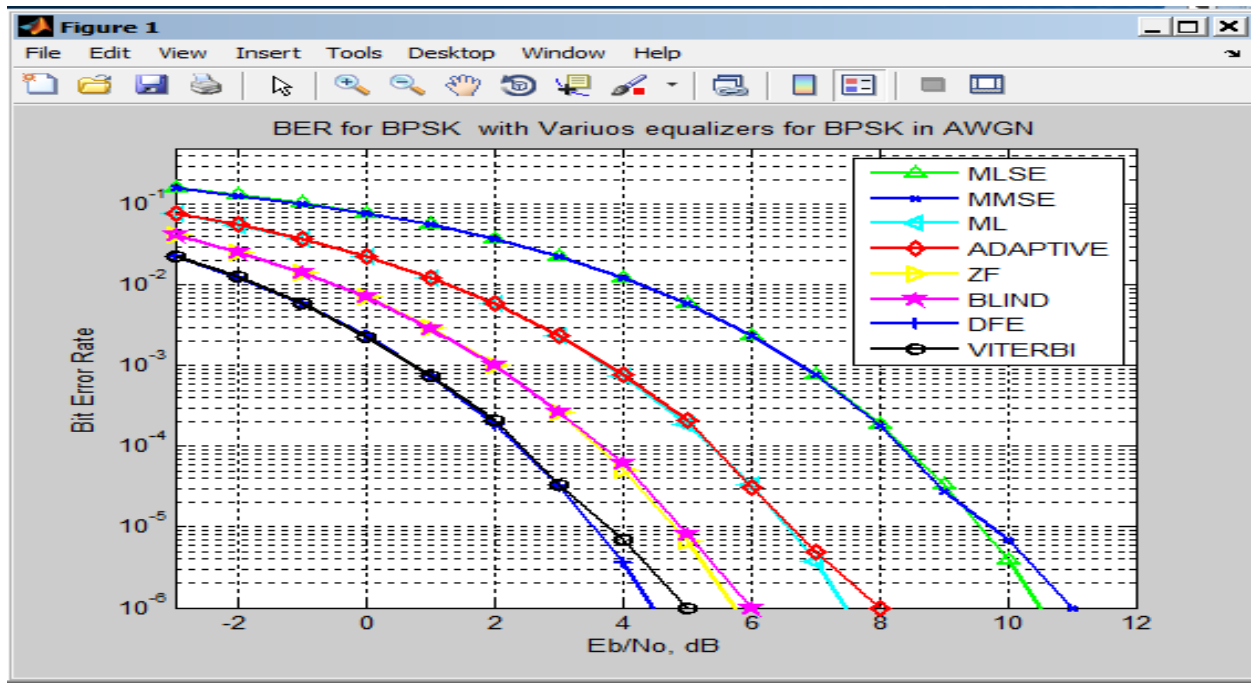

Fig 6. BER for BPSK by using various equalizer in AWGN Channel

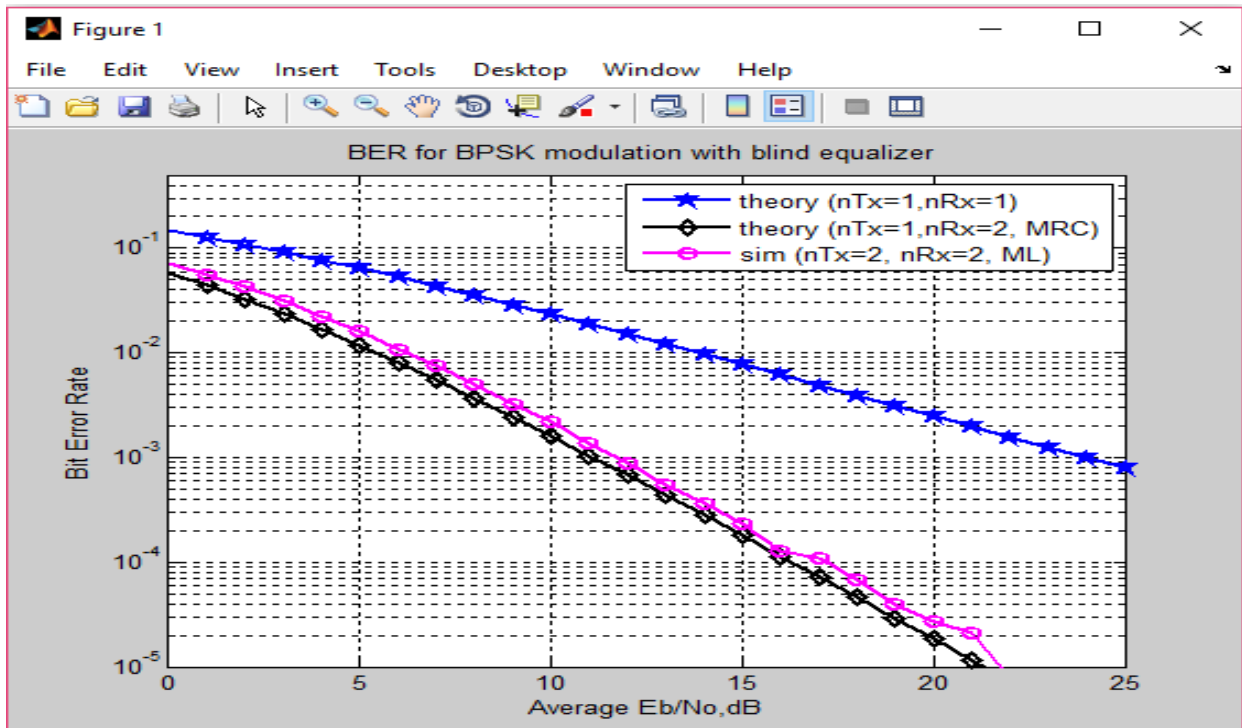

Fig 7. BER for BPSK modulation with blind equalizer

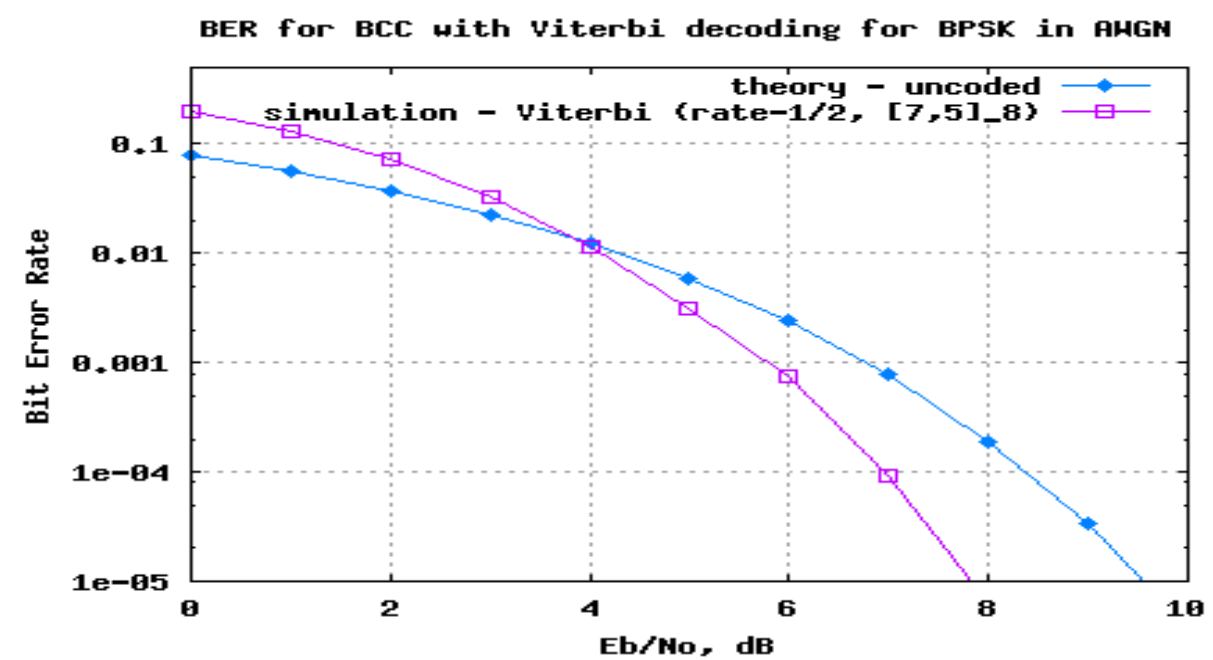

Fig 8. BER for BCC with Viterbi decoding for BPSK in AWGN 
In this work we have analysed the system of wireless communication of OFDM with STBC Coding for Wi-MAX 802.16e standard in MATLAB.

\section{Conclusion}

In this article we presented the technologies like MIMO-OFDM, STBC and WIMAX for current and upcoming wireless communication systems. Simulation results are plotted and comparison of bit error rate (BER) for various equalizers using MATLAB software. From these results we can understand without increasing the communication resources like signal power, transmission bandwidth, high data rate is possible and also coding gain. Hence above technologies are well suited for current $4 \mathrm{G}$ and upcoming $5 \mathrm{G}$ wireless broad band communications.

\section{References}

[1]. G.L. Stuber, J.R. Barry, S.W. McLaughlin, Ye Li, M.A. Ingram and T.G. Pratt, "Broadband MIMO-OFDM wireless communications," Proceedings of the IEEE, vol. 92, No. 2, pp. 271 -294,

[2]. DIG-COMM-BARRY LEEMESSERSCHMITT], Digital Communication: Third Edition, by John R. Barry, Edward A. Lee, and David G. Messerschmitt.

[3]. Shailesh Shankhi, K.Satya Prasad, "Performance Analysis of Channel Estimation Based on MMSE Equalizer in OFDM System", International Journal of Advanced Innovative Research, Vol. 2 Issue 8, ISSN: 2278-7844, pp. no.-155-159.

[4]. H. Jiang and P. A. Wilford, "A hierarchical modulation for upgrading digital broadcasting systems," IEEE Transaction on Broadcasting, vol. 51, pp. 222-229, June 2005.

[5]. P. W. Wolniansky, G. J. Foschini, G. D. Golden and R. A. Valenzuela, "V-BLAST: an architecture for realizing very high data rates over the rich- scattering wireless channel," In Proceeding of International symposium on Signals, Systems and Electronics, pp. 259300 , October 1998.

[6]. IEEE Standard 802.16: A Technical Overview of the Wireless MANTM Ai Interface for Broadband Wireless Access. Available: http://wirelessman.org/docs/02/C80216-02_05.pdf

[7]. 7.WiMAXAvailable:http://ocw.kfupm.edu.sa/user062/COE34202/WiMAX\%20-\%20Wireless\%20to\%20the\%20MAX.pdf

[8]. R. Olexa. implementing 802.11,802.16,and 802.20 wireless network, planning troubleshooting and operations

[9]. Available:http://books.google.co.uk/books?id=qytvAz_VYKQC\&dq=Ron+Olexa,+Implementing $+802.11,+802.16+$ and $+802.20+W$ ireless+Networks, \&printsec=frontcover\&source=bn\&hl=en\&ei=HUp9SvCgM926jAfSsvjwAQ\&sa=X\&oi=book_result\&ct=result $\&$ resnum $=4 \# \mathrm{v}=$ onepage $\& \mathrm{q}=\& \mathrm{f}=\operatorname{true}(\mathrm{pp} \mathrm{xi})$.

[10]. Coherence time. Available: http://en.wikipedia.org/wiki/Coherence_time.

[11]. E. a. C. E. Zhifeng Chen, University of Florida. Performance Analysis of Channel Estimation and Adaptive EqualizationinSlowFadingChannel.Available:http://www.wu.ece.ufl.edu/projects/channelEstimation/documents/Final\%20Report.pd f [1] IEEE. Available: http://en.wikipedia.org/wiki/Ieee 